\title{
Immunoassay Multiplexing on a Complementary Metal Oxide Semiconductor Photodiode Array
}

\author{
Bence Nagy, ${ }^{*}, \oplus$ Mohammed A. Al-Rawhani, ${ }^{\dagger}$ Boon Chong Cheah, ${ }^{\dagger \oplus}$ Michael P. Barrett, \\ and David R. S. Cumming, ${ }^{\dagger}$
}

${ }^{\dagger}$ School of Engineering and ${ }^{\ddagger}$ Institute of Infection, Immunity and Inflammation \& Glasgow Polyomics, College of Medical, Veterinary and Life Sciences, University of Glasgow, Glasgow G12 8LT, United Kingdom

\section{Supporting Information}

ABSTRACT: Scalable immunoassay multiplexing offers a route to creating rapid point-of-care (POC) diagnostics. We present a method for multiplexing immunoassays on the surface of a complementary metal oxide semiconductor (CMOS) sensor array integrated circuit (IC) without the use of physical separators such as wells or channels. Major advantages of using a CMOS sensor array include low mass-manufacturing costs, the possibility to multiplex multiple assays on a single IC, and improved signal when averaging multiple sensors, along with providing a platform where wash steps can be incorporated to maximize selectivity and sensitivity compared to paper based lateral flow immunoassay. The device was able to

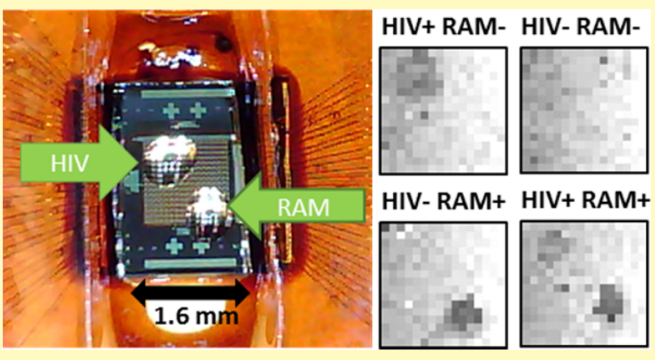
differentiate between samples containing either, neither, or both rabbit anti-mouse (RAM) antibodies and/or anti-HIV gp120 antibodies in serum using a gold-nanoparticle promoted silver enhancement immunoassay. HIV antibody concentrations down to $100 \mu \mathrm{g} / \mathrm{mL}$ were readily detected, which is three times lower than those typically found in infected humans (300-500 $\mu \mathrm{g} /$ $\mathrm{mL}$ ), and the limit of detection was $10 \mu \mathrm{g} / \mathrm{mL}$.

KEYWORDS: immunoassay, CMOS, point-of-care, diagnostics, HIV, silver enhancement

$\mathrm{M}$ any medical tests are invasive, time-consuming, and usually require specialist medical centers. A time-lag therefore ensues while samples are analyzed remotely, and a repeat visit to the medical center is required to discuss outcomes. Furthermore, the ability to home-test for various diseases has improved the ability for rapid diagnosis and implementation of appropriate medical intervention. Home pregnancy testing represents the prime example. ${ }^{1,2}$ A hand-held device not only increases convenience but also offers diagnostics in remote areas where laboratory tests are not locally available.

The usefulness of mobile phones in health care has already been demonstrated. ${ }^{3-9}$ Testing for a multitude of substances that are indicative of disease could be done using devices that link to a smartphone and measure analytes within a drop of blood. The work presented here provides a step in that direction through detection of anti-HIV and rabbit anti-mouse (RAM) antibodies on a hand-held chip-based device (Figure 1). The HIV assay used in this paper has been proven to work with whole blood. ${ }^{10}$

Antibodies are soluble proteins that circulate in blood and bind specifically to foreign molecules (antigens) as part of the adaptive immune response. ${ }^{11}$ Antibodies are frequently exploited in immunoassays for diagnostic purposes as their production is in direct response to the presence of foreign antigens in the host system. In a direct immunoassay, the presence of the disease (antigen) is detected using exogenous antibodies specific to the antigen. In an indirect immunoassay, it is the presence of an antibody to a specific disease (antigen) that is detected (immune response). It is much easier to detect antibodies due to their abundance, ${ }^{12-14}$ although the body needs some time to produce antibodies after being infected (a process termed seroconversion).

Complementary metal oxide semiconductor (CMOS) technology has revolutionized the microelectronic industry, being both low-cost and reliable. In this paper, we demonstrate concurrent, interference-free execution of two optical immunoassays on the surface of a CMOS sensor array chip to detect antibodies targeted to the immunodominant gp120 antigen of $\mathrm{HIV}$, and also antibodies to a second antigen (mouse IgG), demonstrating the potential to develop a multiplexed assay system on the surface of a CMOS sensor array.

Multiple analyte detection is the key to creating a useful health diagnostic device. A proof of concept sandwich immunoassay was developed to demonstrate the functionality of a phone-CMOS platform. In the RAM assay, rabbit antimouse antibodies bind to printed mouse antibodies on the surface of the chip. These antibodies are detected with goldlabeled goat anti-rabbit antibodies.

This assay can be easily modified to detect useful real world analytes such as antibodies to HIV, syphilis, hepatitis, malaria, West Nile virus, Chagas disease, herpes, and Zika. The

Received: December 28, 2017

Accepted: April 13, 2018

Published: April 13, 2018 

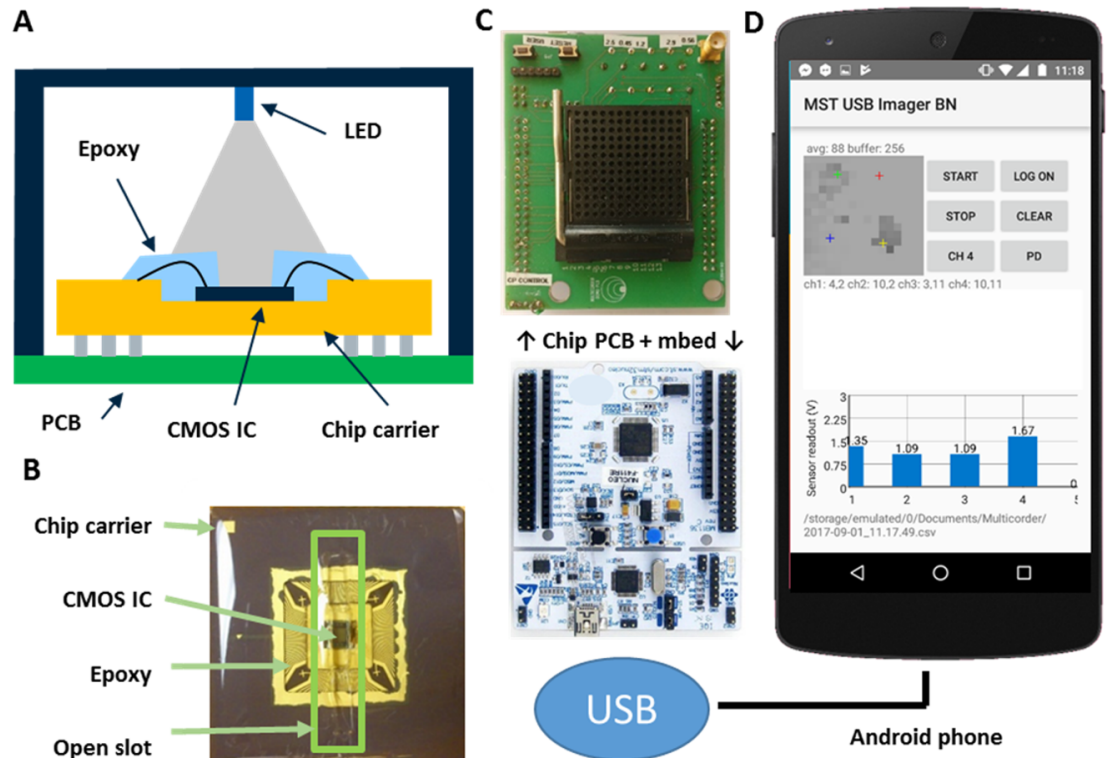

Figure 1. Immunoassay capable smartphone accessory. Images showing the construction of the CMOS chip carrier (A, B) and data acquisition electronics (C) which can be connected to a smartphone or a PC (D). The CMOS chip carrier fits into the socket on the chip PCB. An LED and cover and mounted on top of the chip PCB. On the other side of the chip PCB there are sockets to plug in the mbed (ST-Nucleo) board which handles the data acquisition and communication with the smartphone/PC. The smartphone app is shown displaying the acquired data in visual and numerical form. Colored crosses can be moved around to choose the individual pixels being charted.

technology presented can be adopted to create a single diagnostic chip, for example, that corresponds to all sexually transmitted diseases or pathogens known to cause fever in tropical settings. As it is often hard to ascertain the cause of fever in tropical areas, such a tool would be invaluable. In the assays presented here, droplets of the assay solution were manually printed onto the chip using a polystyrene needle. However, other technologies exist capable of printing much smaller droplets, which in turn would increase the number of assays available on a chip. ${ }^{15,16}$

HIV was chosen for demonstration as it remains a major global public health issue, having claimed more than 35 million lives so far. It is estimated, however, that only $54 \%$ of people with HIV know their status (WHO facts, 2016). Including HIV testing on any personal health diagnostic tool is extremely important. While rapid HIV testing already exists ${ }^{17-19}$ our ultimate aim is to integrate HIV testing with other assays into a single device. Having an HIV test in a personal health machine would be beneficial as it would increase the convenience, privacy and reach of HIV testing. Being easy, low-cost, and anonymous, it could be used in areas where conventional testing is not available.

Immunoassay formats reported in refs 10 and 20 allow multiple assays to be performed on the surface of a single chip where different proteins can be positioned with precision on the chip surface. The assay then uses changes in the absorption of light applied to the surface allowing detection with a photodiode. The sensitivity of the silver-amplified immunoassay reported in ref 10 rivals that of the gold standard laboratorybased HIV ELISA tests, but also includes antigens capable of detecting seroconversion to syphilis. A polystyrene microfluidic cassette, with individual detectors for each assay, thus offers a system that can detect HIV and syphilis in a drop of blood, using a smartphone accessory. While proving the ability to make multiple assays on a single chip, scaling up the total number of possible assays is restricted by engineering requirements for the microfluidic cassettes.

A major advantage of using silicon CMOS technology is that it readily allows for miniaturization so that multiple sensors can be cointegrated at low cost. Furthermore, the ability to integrate electronic functionality such as signal amplification and data multiplexing from an array of sensors enables new multiple assay technologies to be realized. While CMOS possesses many of the required attributes of a biosensor technology, a certain amount of post-processing (back-end of line) is necessary, and we show how a readily available epoxy based photoresist used for microelectro-mechanical systems, SU-8, can be exploited to facilitate assay immobilization technology, making multiple assays on a single chip possible. The combination of CMOS and immobilization technologies allows qualitative analysis from multiple assays simultaneously with the sensitivity needed to measure physiological levels of antibodies found for many diseases in human blood.

The CMOS array presented has 256 sensors. With sufficiently advanced inkjet printing technology, each couple of sensors can be functionalized to perform different immunoassays. Even with the manual printing technique used in this work, up to six droplets were printed on a single chip with functioning antibodies. However, there are only three distinct biological assays presented here (including the control).

\section{RESULTS AND DISCUSSION}

Instrumentation and Measurement System. An ARM mbed data acquisition platform was assembled that can be connected to a smartphone/PC via USB or Bluetooth (Figure 1C-D). An Android app was created to evaluate the usefulness of a smartphone connected blood testing device.

Once the chip was packaged and processed and the immunoassay was executed, the chip was inserted into a PGA ZIF socket attached to a custom printed circuit board (Newbury Electronics, UK) designed to connect the chip 
carrier to the COTS mbed board (NUCLEO-F334R8, STMicroelectronics).

An external power supply was used to power the chip during all experiments presented. However, USB OTG was used to power and communicate with the entire system during smartphone connected trials.

Initial experiments to verify the chip and the assay were conducted with the platform attached to a PC, but as the technology matured we connected the platform to a smartphone to fulfill our objective. The USB serial interface of the mbed board was used for communication. First, the PC/ smartphone sends a command byte to the mbed, and then the mbed sends back 256 bytes, each corresponding to a pixel readout. The bytes are counted to make sure no data loss occurred and then presented on the screen. Also, all the data is streamed to permanent storage using the CSV file format or, in the case of LabView, the National Instruments TDMS file format. Reading an entire frame in this way takes less than 100 ms. The serial bus is set to run at 115200 baud.

The mbed measures the voltage output of each pixel with the use of its analog to digital converter (ADC). It iterates through all the pixels in the array by outputting their digital addresses in sequence. Once a valid address is presented, the voltage output of the corresponding pixel appears on the analog output pin. It sends all the acquired data through its serial interface.

The USB OTG connected android acquisition app developed surpassed the PC based LabView VI in both stability and speed. Another advantage of a wired (not Bluetooth) smartphone accessory is that it does not need its own power source. The android app is available for download in the Google Play store under "Multicorder USB".

Experimental Results. Antibody detection was chosen over antigen detection in this study (Figure 2) because of the relative ease of detection of antibodies in seroconverted blood. ${ }^{12,13}$ However, sandwich assays using a bait antibody to capture proteins from serum, which can then be detected using a second antibody, this time gold nanoparticle labeled, would be equally useful with this same setup.

A positive response results in the appearance of dark spots at positions where the corresponding proteins were printed. Otherwise, the image is homogeneous. Spectral transmission of the silver spots was measured with a spectrophotometer to aid in the choice of an LED emitting light at the correct wavelength, although there was no significant practical advantage of using one color over the other. The system we present is analogous to an ELISA type assay but miniaturized and amenable to simple electronic readout using a smartphone. The sensor chips were fully characterized before performing the experiments.

The sensor array was functionalized by the addition of droplets containing different proteins, including the HIV gp120 protein and the mouse IgG (Figure $3 \mathrm{~A}, \mathrm{~B}$ ). The droplets were printed manually with a polystyrene needle. The antigens dissolved in the droplets adhered to the surface via direct physisorption. Once the chip was functionalized and washed, the entire chip was treated with BSA blocking buffer to avoid nonspecific antibody binding. Antibodies specific to these proteins were then added and nonspecifically bound antibody washed from the chip surface. Nanogold-labeled secondary antibodies were then added to bind to those primary sera and these caused the precipitation of added silver within their direct vicinity when this later reagent was added. The precipitation masks photons from the LED from reaching the photodiode

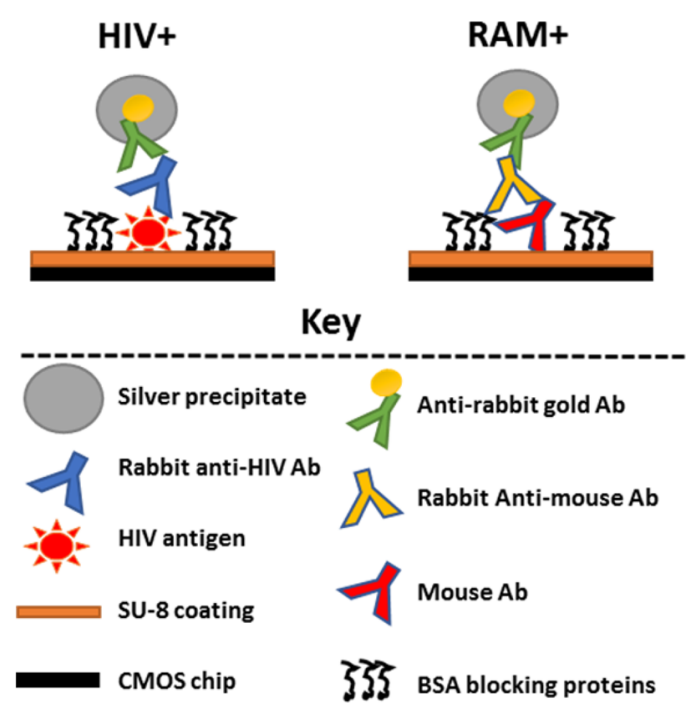

Figure 2. Drawings showing the biochemical construction of the HIV and the RAM assay. These assays are built step by step on the surface of the chip with washes in between each step. The aim is to detect the presence of HIV antibodies and/or anti-mouse rabbit (RAM) antibodies in rabbit serum independent of each other, concurrently, in the same sample solution. [1] The chip is covered with SU-8 photoresist. [2] Two droplets are printed on the surface of the chip: one containing HIV gp120 antigen and one containing mouse IgG (both in bicarbonate buffer, $10 \mu \mathrm{g} / \mathrm{mL}$ ). Next, the chip is covered with [3] BSA blocking buffer (PBS, 1\%), [4] rabbit serum containing either, both, or neither anti-HIV gp120 rabbit antibodies and anti-mouse rabbit (RAM) antibodies (this is the analyte, $100 \mu \mathrm{g} / \mathrm{mL}, 65 \%$ rabbit serum, PBS), [5] gold conjugated anti-rabbit antibody (A520 = 0.1), [6] silver nitrate/hydroquinone solution (citrate buffer).

detectors at the CMOS surface and the increase in absorbance is detected electronically.

Once the assay was complete, the chip was dried and inserted into the measurement system and the incident light on the printed areas was measured and plotted with data from the negative control areas which remained transparent. Experiments were repeated at least three times on different devices with similar results. Run-to-run variation that can occur with the illumination system in our prototype was compensated for in the data presentation by graphing the difference between control and active areas over many experiments (Figure 3C).

Figure 3D shows the results of positive and negative HIV and rabbit anti-mouse (RAM) tests in serum printed on the surface of the SU-8 coated chip. For each of these experiments, two droplets were printed on the surface: one for the HIV and one for the RAM assay. The printed surfaces only turned dark if the test was positive; that is, antibody specific for the target antigen was present in the serum. Negative control (HIV-/RAM-) experiments were executed with the same analyte solution (rabbit serum) but without the addition of the target antibody.

The data clearly demonstrates that the system can make two separate assay measurements concurrently on the same chip, without interference. The data we obtained was of sufficiently high quality to enable automated electronic threshold analysis to determine the diagnostic outcome.

It can be seen that the voltage difference between positive and negative control areas was $0.16 \mathrm{~V}$ for HIV and $0.36 \mathrm{~V}$ for $\mathrm{RAM}$, on average. The lowest difference we observed for a positive HIV assay was $0.1 \mathrm{~V}$ while the largest difference between negative control areas was $0.07 \mathrm{~V}$. The achievable 

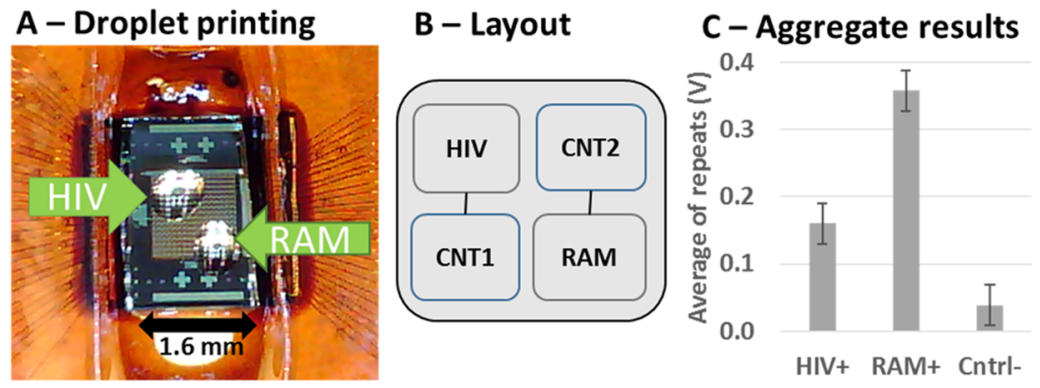

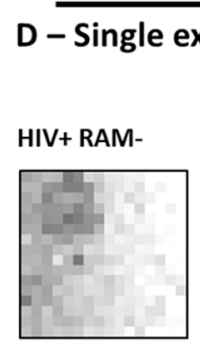

HIV- RAM+

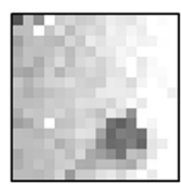

HIV+ RAM

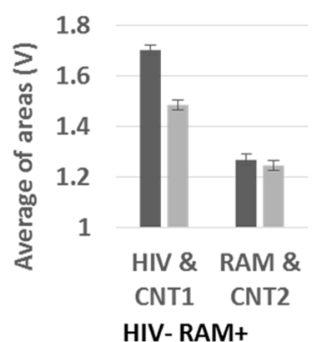

HIV+ RAM+

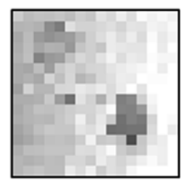

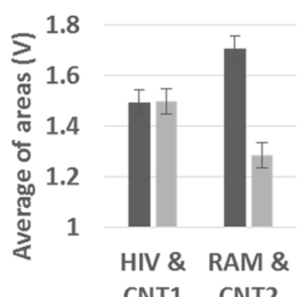
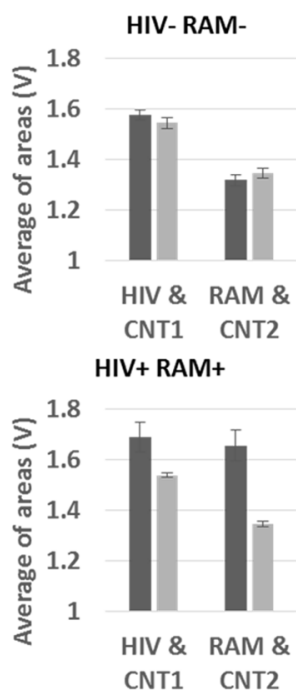

Figure 3. Multiplexed HIV/RAM assay results in serum on CMOS chip with SU-8 surface. (Serum was diluted with water 65:35, data acquisition system was connected to a smartphone.) (A) Typical size and position of printed droplets. HIV gp-120 antigen solution on the top left, mouse IgG solution on the bottom right. (B) The epoxy walls and the misalignment of the illumination create a background gradient signal. To correct for this, each printed droplet has a corresponding control area in the same column. While measurements of the chip before carrying out the assay provide some control data, by measuring control pixels on the chips after the assay is done more faithfully enables accurate interpretation of the data. (C) The difference in the printed and control area signals can be used to chart different experiments (executed on different devices) together. This differential signal is averaged over 12 experiments and charted ( 3 repeats of HIV+, RAM+, combination, and negative control experiments). (D) Images and bar charts displaying data acquired from the chip. There are four possible different outcomes of HIV/RAM multiplexed assay. If the rabbit serum sample contains HIV rabbit antibodies, the HIV area develops a dark spot, if it contains rabbit anti-mouse (RAM) antibodies, then the RAM area develops a dark spot. Typically 10-30 pixels are averaged to calculate the signal level of a printed/control area. The contrast of these grayscale images was enhanced for illustration purposes.

electronic limit of detection is set by the supply voltage ( $3.3 \mathrm{~V}$ ) and the number of bits of the analog to digital converter (8) to be $0.01 \mathrm{~V}$. The data are therefore considerably larger than the errors we determined from the system; hence, we estimate that any assay that produces a voltage difference of more than 0.09 $\mathrm{V}$ can be considered positive.

$$
\begin{aligned}
& \text { Result_HIV }=\operatorname{avg}(\text { HIV pixels })-\operatorname{avg}(\mathrm{CNT} 1 \text { pixels }) \\
& \text { Result_RAM }=\operatorname{avg}(\text { RAM pixels })-\operatorname{avg}(\mathrm{CNT} 2 \text { pixels }) \\
& \text { Error }=\frac{\sigma}{\sqrt{ } N}
\end{aligned}
$$

The results of Figure 3D were obtained by using the averaging capabilities from multiple independent pixels provided by the photodiode sensor array: antigen droplets were printed on the surface such that each droplet covered $N=$ 9-30 sensors (Figure 3B). The signal standard deviation is reduced by $\sqrt{ } N$, where $N$ is the number of sensors. When the data was acquired the location of these pixel clusters were known, using optical microscopy, and used to produce an averaged result. Negative control areas were also chosen and, again, the average signal from the cluster of pixels was taken. Antibodies tended to concentrate at the edges of the droplet, and so the edges became darker than the middle (coffee ring effect $\left.{ }^{21}\right)$. By averaging the entire area covered by the droplets, false negative results could be avoided. The analyte (HIV gp120 antibody, anti-mouse rabbit antibody) concentration detected was $100 \mu \mathrm{g} / \mathrm{mL}$ in rabbit serum (65\%) diluted with PBS. The detection part of the assay took 45 min (analyte incubation, gold labeled antibody incubation, silver development).

In order to characterize the assays in depth, the parameter space for all of the reagents and the reaction time was studied. A complementary set of experiments was conducted on polystyrene, the same material that is used for ELISA plates, the current gold standard in immunoassays. Polystyrene slides were placed on top of the CMOS chip. The CMOS chip was masked by a cover with a small hole in the middle, focusing light to the area of interest in the polystyrene slide. The entire photodiode array was averaged in this case, so the chip functioned as an ELISA plate reader. The assay referred to as positive control (rabbit IgG) assay is a simpler version of the RAM assay: gold conjugated anti-rabbit IgG binds to the printed rabbit IgG on the surface. 

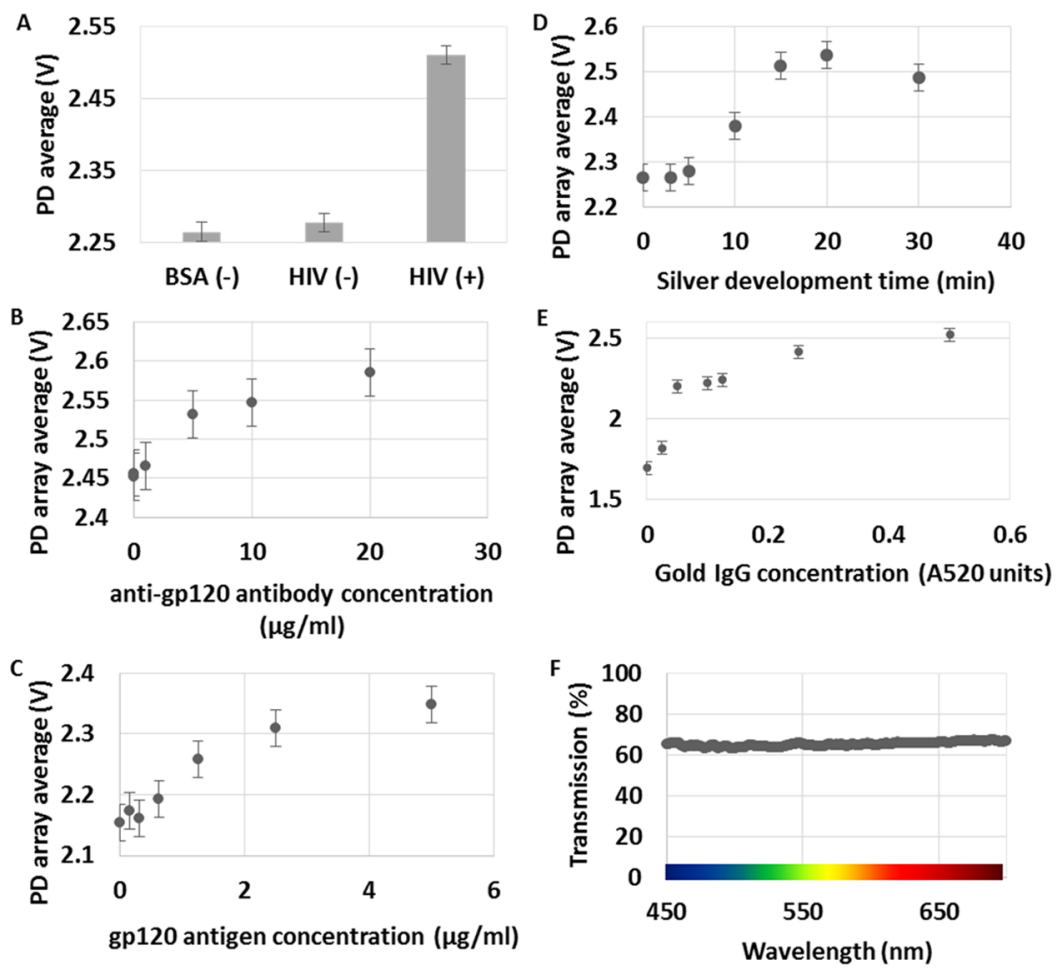

Figure 4. Assay characteristics on polystyrene (A-E) and glass (F). (Data acquisition system was connected to a PC.) (A) Result of two negative control assays and an HIV+ assay were measured by the photodiode array. The HIV- assay is an HIV assay where the anti-gp120 antibody was substituted with IgG solution to simulate a negative sample, whereas the BSA- assay lacks the HIV capture protein (gp120) on the surface, so the anti-gp120 antibody in the analyte has nothing to bind to. (B) HIV antibody concentration of the HIV+ assay was varied. (C) Printed HIV antigen concentration of the HIV+ assay was varied. (D) Silver development time of the positive control assay was varied. (E) Concentration of the gold conjugated antibody solution of the positive control assay was varied. (A520 is a measure of light absorbance at $520 \mathrm{~nm}$ which is directly proportional to the concentration of gold conjugated antibodies). (F) Transmission spectrum of a silver spot developed on an SU-8 covered glass

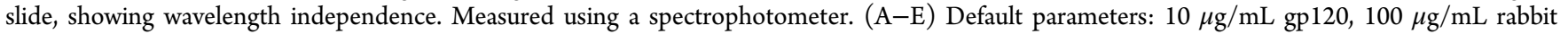
anti-gp120, 15 min silver development.

Figure 4A shows the outcome of the HIV assay when the antibodies in the analyte were present (HIV+), absent (HIV-), and when the gp120 capture protein on the surface is absent (BSA-). Figure 4B,C shows the outcome of the HIV assay when the concentration of the printed HIV gp120 antigen or the rabbit anti-gp120 antibody was varied. These graphs can be used to determine the minimum concentration of printed antigen required, as well as the minimum detectable concentration of anti-gp120 antibody. Figure 4B represents 2 separate runs of experiments with 4 droplets printed for each data point. Figure $4 \mathrm{C}$ represents a single run of experiments with 2 droplets printed for each data point (since this assay was repeated with fixed concentrations many times, no further repeats were deemed necessary).

Figure 4D,E shows results of the positive control (rabbit $\operatorname{IgG}$ ) assays with two parameters varied. These experiments serve two purposes. They demonstrate the effects that reagent concentrations and development time have on the outcome. They also indicate the minimum required concentrations and time needed to detect antibodies.

Figure 4D shows the outcome of the positive control experiment as the silver development time is varied to determine the minimum amount of time required for the silver to develop on positive control areas. The assay needs at least $10 \mathrm{~min}$ to produce a significant difference between positive and negative control areas. Accordingly, 15 min was chosen as the minimum amount of development time that guarantees a successful positive control. Figure $4 \mathrm{D}$ represents 5 droplets for each data point acquired from two separate runs of the experiment.

Figure $4 \mathrm{E}$ shows how the outcome of a positive control experiment changes when varying the secondary gold conjugated antibody concentration. The assay starts working at a dilution of $1: 100\left(A_{520}=0.05\right)$ and continues to improve as the concentration goes up. A 1:50 dilution $\left(A_{520}=0.1\right)$ was chosen to ensure sufficient antibody is present to indicate a positive result without using an excessive amount of reagent, which has an economic benefit. Figure 4E represents a single run of assays, 3 droplets were printed for each data point (since this assay was repeated with fixed concentrations many times, no further repeats were deemed necessary).

\section{MATERIALS AND METHODS}

CMOS Sensor Chip. The sensor chip used in this work incorporates an array of $16 \times 16$ pixels. Each pixel incorporating a $\mathrm{p}-\mathrm{n}$ junction photodiode with three transistors for readout. ${ }^{22}$ The chip uses a global shutter scheme for exposure and a scanning scheme for acquisition where one 4-bit row decoder and one 4-bit column decoder are integrated outside the active area.

The chip measures $3.4 \mathrm{~mm} \times 3.6 \mathrm{~mm}$ and was fabricated using the AMS $0.35 \mu \mathrm{m}$ CMOS process. The chip was designed so its wire bonds are only on two sides of the chip. This allows easy access to the surface of the chip. For our work we used a custom designed photodiode array since commercially available devices are packaged in such a way that they inhibit experimentation. In the future, commercial chips could be used. 
Chip Packaging. The bond wires and parts of the chip were protected from aqueous reagents and cleaning solutions (Figure 1A,B). The chip was mounted on a chip carrier using epoxy (EPO-TEK $\mathrm{H} 74$ ). The epoxy was cured at $150{ }^{\circ} \mathrm{C}$ for $10 \mathrm{~min}$. The chip was then wire bonded to the chip carrier. Polydimethylsiloxane (PDMS) cuboids were used to cover the chip sensor area and to make a channel leading to the edges of the chip carrier. Next, epoxy resin (EPO-TEK $302-3 \mathrm{M}$ ) was applied to the bond wires and bond pads. The PDMS cuboids prevented epoxy from covering the surface of the sensor array. The epoxy was cured at room temperature for $24 \mathrm{~h}$. Then the chip was covered with SU-8 3005 photoresist and spun at $1500 \mathrm{rpm}$ for $30 \mathrm{~s}$. The channel leading to the edge of the chip carrier allows the excess resist to escape the surface of the chip while spinning. The resist was cured with heat and UV radiation $\left(90^{\circ} \mathrm{C}\right.$ oven for $1 \mathrm{~h}, 200 \mathrm{~mJ} / \mathrm{cm}^{2}$ UV). The resulting layer of SU-8 was approximately $10 \mu \mathrm{m}$ thick.

Once the immunoassay was executed on the surface of the chip, the SU-8 resist layer, along with any biological material was stripped using piranha solution (3:1 sulfuric acid and hydrogen peroxide, $3-15 \mathrm{~min}$ ). Some chips were reused (with a fresh coat of SU-8) until defects were detected. As a medical device these chips are meant to be single use, so the parameters of reusability were not investigated further.

Chemicals and Reagents. Phosphate buffered saline (PBS), bovine serum albumin (BSA), bicarbonate buffer, rabbit IgG, antimouse rabbit $\mathrm{IgG}$, mouse $\mathrm{IgG}$, rabbit serum, rabbit anti-gp 120 antibody, $10 \mathrm{~nm}$ gold conjugated goat anti-rabbit IgG, silver nitrate, citric acid, trisodium citrate dehydrate, and hydroquinone were obtained from Sigma-Aldrich Co., UK. Recombinant HIV gp120 antigen was obtained from Fisher Scientific UK Ltd.

HIV gp120 antigen solution was prepared using $10 \mu \mathrm{g} / \mathrm{mL}$ gp120 in bicarbonate buffer. Rabbit IgG solution was prepared using $10 \mu \mathrm{g} / \mathrm{mL}$ rabbit IgG in bicarbonate buffer. 1\% BSA blocking buffer was prepared using PBS. The HIV/RAM test antibody solution was made up of 100 $\mu \mathrm{g} / \mathrm{mL}$ rabbit anti-gp120 and/or anti-mouse rabbit antibody in PBS and rabbit serum (35-65\%). Gold conjugated anti-rabbit IgG stock solution was diluted 1:50 using 3\% BSA solution. Silver developer solution was prepared fresh before use containing $500 \mu \mathrm{g} / \mathrm{mL}$ silver nitrate and $17 \mathrm{mg} / \mathrm{mL}$ hydroquinone using citrate buffer. Citrate buffer was prepared containing $2.35 \%$ citric acid and $2.55 \%$ trisodium citrate. All solutions were prepared with Millipore water.

Immunoassay Procedure. The CMOS chip was coated with 10$\mu$ m-thick SU-8 photoresist. HIV antigen solution and mouse IgG solution was spotted on the surface of the chip using polystyrene inoculation needles (Fisher Scientific Ltd.). The spots were incubated at room temperature in a sealed, humid container for an hour. The chip was rinsed with PBS and water, covered with blocking buffer, and incubated for an hour. The chip was then rinsed and covered with HIV/RAM test antibody serum solution for $10 \mathrm{~min}$, then rinsed again and covered with gold conjugated antibody solution for $10 \mathrm{~min}$. After rinsing, the chip was covered with developer solution and incubated for $25 \mathrm{~min}$ under a dark cover. The resulting structure is illustrated in Figure 2. Finally, the chip was rinsed in water, dried, and inserted into the data acquisition system. A blue LED (VLMB1300-GS08) was used to illuminate the surface of the chip. The brightness was adjusted to achieve optimal illumination (PD output 1-2.5 V, different chips needed different brightness). Each photodiode in the array produced a voltage depending on the amount of light that reached it.

\section{CONCLUSION}

Two localized silver staining assays that function essentially as an ELISA presented in ref 17 were successfully integrated and multiplexed on the surface of a CMOS sensor array. The advantages of running assays directly on the surface of a sensor array are manifold. The average of multiple sensors can be used to reliably evaluate a single assay, and, crucially, multiple assays can be executed concurrently on the surface of a single chip. The approach also allows wash steps that increase sensitivity and specificity when compared to popular paper based lateral flow immunoassays. This is the first step in creating a mass- manufacturable hand-held point-of-care (POC) personal diagnostic tool capable of performing all necessary medical tests in one device.

The results presented show that the positive HIV assay, as well as the RAM assay, produce a signal comparable to the positive control. Furthermore, the assays on top of an SU-8 covered CMOS chip produce similar signals to those generated using polystyrene plates, the current gold standard for ELISA assays. The concentration of the HIV antibody used here is three times lower than reported in typical human infections $(300-500 \mu \mathrm{g} / \mathrm{mL}){ }^{11}{ }^{1}$ hence, this assay would be suitable to detect HIV antibodies in human blood. It is also demonstrated that multiple assays can be performed concurrently in the same solution without these assays interfering with each other. Here, we have shown that antibodies specific to HIV gp120 and antibodies specific to mouse IgG are simultaneously identified. Next we will extend the array of detected antigens to a range of other pathogens.

Signal strength can also be controlled by varying parameters such as reagent concentration and development time, and as we scale the device to include additional pathogen detection immunoassyas, we will optimize conditions appropriately.

Both a PC-based and a smartphone-based data acquisition system were designed and evaluated. The USB OTG connected smartphone app is excellent for reliably acquiring high-speed data surpassing the PC based system in both speed and reliability. This proves that the concept of a complex hand-held diagnostic device is only a few steps away.

\section{ASSOCIATED CONTENT}

\section{S Supporting Information}

The Supporting Information is available free of charge on the ACS Publications website at DOI: 10.1021/acssensors.7b00972. The entire data set is also available at http:// dx.doi.org/10.5525/gla.researchdata.602

$$
\text { Supplementary data (PDF) }
$$

\section{AUTHOR INFORMATION}

\section{Corresponding Authors}

*E-mail: B.Nagy.1@research.gla.ac.uk.

*E-mail: David.Cumming.2@glasgow.ac.uk.

ORCID

Bence Nagy: 0000-0003-4974-7423

Boon Chong Cheah: 0000-0002-2580-4094

\section{Author Contributions}

The manuscript was written through contributions of all authors. All authors have given approval to the final version of the manuscript. M.A.A. designed the CMOS chip, B.C.C. developed chip packaging techniques, B.N. performed and adapted immunoassays, developed immobilization, chip recycling and packaging techniques, collected and analyzed the data and created the app. M.A.A., B.C.C., and B.N. created LabView data collection applications.

\section{Notes}

The authors declare no competing financial interest.

\section{ACKNOWLEDGMENTS}

We would like to thank the funders: EPSRC grant no. EP/ K021966/1 (United Kingdom). M.P.B. is also supported by a core grant to the Wellcome Centre for Molecular Parasitology $104111 / \mathrm{Z} / 14 / \mathrm{Z}$. We thank the entire MST research group at 
the University of Glasgow, especially C. Martin for training and advice, and N. Keegan and C. Dale at Newcastle University for advice.

\section{ABBREVIATIONS}

POC, point-of-care; CMOS, complementary metal oxide semiconductor; IC, integrated circuit; HIV, human immunodeficiency virus; RAM, rabbit anti-mouse; ELISA, enzymelinked immunosorbent assay; LED, light emitting diode; WHO, World Health Organization; PBS, phosphate buffered saline; BSA, bovine serum albumin; CSV, comma separated values; $\mathrm{PCB}$, printed circuit board; PGA, pin grid array; ZIF, zero insertion force; UV, ultraviolet; PDMS, polydimethylsiloxane; COTS, commercial off-the-shelf

\section{REFERENCES}

(1) Nettleman, M. D.; Brewer, J. R; Ayoola, A. B. Self-Testing for Pregnancy Among Women at Risk: A Randomized Controlled Trial. Am. J. Prev. Med. 2009, 36 (2), 150-153.

(2) Entry Into Prenatal Care - United States, 1989-1997; http:// www.cdc.gov $/ \mathrm{mmWr} /$ preview $/ \mathrm{mmwrhtml} / \mathrm{mm} 4918 \mathrm{a} 1 . h \mathrm{~m}$.

(3) Wei, Q.; Qi, H.; Luo, W.; Tseng, D.; Ki, S. J.; Wan, Z.; Göröcs, Z.; Bentolila, L. A.; Wu, T.-T.; Sun, R.; et al. Fluorescent Imaging of Single Nanoparticles and Viruses on a Smart Phone. ACS Nano 2013, 7 (10), 9147-9155.

(4) Zurovac, D.; Sudoi, R. K.; Akhwale, W. S.; Ndiritu, M.; Hamer, D. H.; Rowe, A. K.; Snow, R. W. The Effect of Mobile Phone TextMessage Reminders on Kenyan Health Workers' Adherence to Malaria Treatment Guidelines: A Cluster Randomised Trial. Lancet 2011, 378 (9793), 795-803.

(5) Freifeld, C. C.; Chunara, R.; Mekaru, S. R.; Chan, E. H.; KassHout, T.; Ayala Iacucci, A.; Brownstein, J. S.; et al. Participatory Epidemiology: Use of Mobile Phones for Community-Based Health Reporting. PLoS Med. 2010, 7 (12), e1000376.

(6) Chin, C. D.; Cheung, Y. K.; Laksanasopin, T.; Modena, M. M.; Chin, S. Y.; Sridhara, A. A.; Steinmiller, D.; Linder, V.; Mushingantahe, J.; Umviligihozo, G.; et al. Mobile Device for Disease Diagnosis and Data Tracking in Resource-Limited Settings. Clin. Chem. 2013, 59 (4), 629-640.

(7) Oncescu, V.; Mancuso, M.; Erickson, D. Cholesterol Testing on a Smartphone. Lab Chip 2014, 14 (4), 759-763.

(8) Nemiroski, A.; Christodouleas, D. C.; Hennek, J. W.; Kumar, A. A.; Maxwell, E. J.; Fernández-Abedul, M. T.; Whitesides, G. M. Universal Mobile Electrochemical Detector Designed for Use in Resource-Limited Applications. Proc. Natl. Acad. Sci. U. S. A. 2014, 111 (33), 11984-11989.

(9) Lee, D.-S.; Jeon, B. G.; Ihm, C.; Park, J.-K.; Jung, M. Y. A Simple and Smart Telemedicine Device for Developing Regions: A PocketSized Colorimetric Reader. Lab Chip 2011, 11 (1), 120-126.

(10) Laksanasopin, T.; Guo, T. W.; Nayak, S.; Sridhara, A. A.; Xie, S.; Olowookere, O. O.; Cadinu, P.; Meng, F.; Chee, N. H.; Kim, J.; et al. A Smartphone Dongle for Diagnosis of Infectious Diseases at the Point of Care. Sci. Transl. Med. 2015, 7 (273), 273re1.

(11) Greenfield, E. A. Antibodies: A Laboratory Manual, 2nd ed.; Cold Spring Harbor Laboratory Press, 2014.

(12) Binley, J. M.; Klasse, P. J.; Cao, Y.; Jones, I.; Markowitz, M.; Ho, D. D.; Moore, J. P. Differential Regulation of the Antibody Responses to Gag and Env Proteins of Human Immunodeficiency Virus Type 1. J. Virol. 1997, 71 (4), 2799-2809.

(13) Rychert, J.; Strick, D.; Bazner, S.; Robinson, J.; Rosenberg, E. Detection of HIV gp120 in Plasma during Early HIV Infection Is Associated with Increased Proinflammatory and Immunoregulatory Cytokines. AIDS Res. Hum. Retroviruses 2010, 26 (10), 1139-1145.

(14) Klasse, P. J.; Moore, J. P. Is There Enough gp120 in the Body Fluids of HIV-1-Infected Individuals to Have Biologically Significant Effects? Virology 2004, 323 (1), 1-8.
(15) Irvine, E. J.; Hernandez-Santana, A.; Faulds, K.; Graham, D. Fabricating Protein Immunoassay Arrays on Nitrocellulose Using DipPen Lithography Techniques. Analyst 2011, 136 (14), 2925.

(16) Joh, D. Y.; Hucknall, A. M.; Wei, Q.; Mason, K. A.; Lund, M. L.; Fontes, C. M.; Hill, R. T.; Blair, R.; Zimmers, Z.; Achar, R. K.; et al. Inkjet-Printed Point-of-Care Immunoassay on a Nanoscale Polymer Brush Enables Subpicomolar Detection of Analytes in Blood. Proc. Natl. Acad. Sci. U. S. A. 2017, 114 (34), E7054-E7062.

(17) Plate, D. K. Group, on behalf of the R. H. T. E. W. Evaluation and Implementation of Rapid HIV Tests: The Experience in 11 African Countries. AIDS Res. Hum. Retroviruses 2007, 23 (12), 14911498.

(18) Delaney, K. P.; Branson, B. M.; Uniyal, A.; Phillips, S.; Candal, D.; Owen, S. M.; Kerndt, P. R. Evaluation of the Performance Characteristics of 6 Rapid HIV Antibody Tests. Clin. Infect. Dis. 2011, 52 (2), 257-263.

(19) Kagulire, S. C.; Opendi, P.; Stamper, P. D.; Nakavuma, J. L.; Mills, L. A.; Makumbi, F.; Gray, R. H.; Shott, J. P.; Serwadda, D.; Reynolds, S. J. Field Evaluation of Five Rapid Diagnostic Tests for Screening of HIV-1 Infections in Rural Rakai, Uganda. Int. J. STD AIDS 2011, 22 (6), 308-309.

(20) Lackie, P. M. Immunogold Silver Staining for Light Microscopy. Histochem. Cell Biol. 1996, 106 (1), 9-17.

(21) Deegan, R. D.; Bakajin, O.; Dupont, T. F.; Huber, G.; Nagel, S. R; Witten, T. A. Capillary Flow as the Cause of Ring Stains from Dried Liquid Drops. Nature 1997, 389 (6653), 827-829.

(22) Fossum, E. R. CMOS Image Sensors: Electronic Camera-on-aChip. IEEE Trans. Electron Devices 1997, 44 (10), 1689-1698. 